\title{
Induction of protective immunity to mycobacterial infections
}

\author{
P H LAGRANGE \& B HURTREL \\ Institut Pasteur, Paris, France
}

\begin{abstract}
Introduction
Mycobacterial infections and diseases through the world have focused much attention on the often exaggerated immune response which has been investigated from the standpoint of immunity to disease, diagnosis, analysis of the basic immune responses and immunotherapy of several unrelated diseases. However there are few diseases that so effectively express essentialy the entire spectrum of immune responsiveness as leprosy and, to a lesser extent, tuberculosis. For other mycobacterial diseases apart from leprosy and tuberculosis, infection is far less apt to lead to disease in a normal immunocompetent individual. Opportunism in compromised host may be one factor and recent examples in AIDS patients give concrete example of such disseminated mycobacteriosis (1). Many recent investigative efforts are directed towards the explanation of the nature of the immune responsiveness variability seen in leprosy (2) and in tuberculosis (3). Some progress has been made in elucidating the role of suppressive or helper influences of cells, or their mediators, and in reconstituting the immune defects. However, many parameters are involved, concerning dependent and independent variables in the different assays used, and need to be clearly identified in order to clarify their exact role, which they play in induction, expression and regulation of the immune protection induced after a natural or experimental infection.
\end{abstract}

\section{Immune responses after mycobacterial infections}

Chronic mycobacterial infections develop through an immunological spectrum that ranges from a state of high resistance (protection) to an opposite extreme of low resistance and anergy. However, expression of high resistance can be associated in certain individuals with diseases expressing severe immunological reactions such as tuberculoid leprosy, upgrading reactions in borderline leprosy, or increased pulmonary lesions at the onset of efficient chemotherapy in pulmonary tuberculosis. Both leprosy and tuberculosis encompass the entire gradient of the immune spectrum, which may also be seen with other mycobacterial diseases in man and in animals (i.e. Johne's disease in cattle).

In susceptible hosts, inoculation of pathogenic mycobacteria through its own multiplication in macrophages induces specific and non-specific immune responses. Undoubtedly specific antibodies are produced in response to natural or experimental mycobacterial infections, but there is evidence that they play no role in host protection (4), even if some reports have shown that passive immunization with specific antimycobacteria sera in BCG infected mice to a certain extent promote the growth of bacilli in the spleen (5). The mechanism of such an enhancement has not been fully explored but does not seem to involve a higher bacterial uptake of the spleen. However, since it occurred only after a low inoculum, it might involve a particular distribution of the mycobacteria in subpopulations of macrophages which 
are either less able to kill the mycobacteria or more able to promote the growth of the bacilli in absence of the T-cell dependent response, as measured by the DTH reaction (6). Moreover, no data were given concerning the effects of such passive transfer on the acquired resistance of the infected mice.

Thus, most of our knowledge about immunological mechanisms controlling mycobacterial diseases concerns only cell-mediated immunity in experimental or human tuberculosis and leprosy. The specific responses are well characterised by mechanisms which prevent further multiplication and spreading of mycobateria. These events, called macrophage activation and granuloma formation, are specific in their induction but are non-specific in their expression, since they are locally able to inhibit growth and dissemination of other obligate or facultative unrelated intracellular multiplying microorganisms (7). Several excellent reviews have appeared during the past few years on the immunology of mycobacterial infections (8-12); from these and other relevant literature, it can be concluded that the specificity of the immune response to pathological or non-pathological mycobacteria has been shown to be mediated by thymus-dependent lymphocytes (T lymphocytes). Specific, uncommited $\mathrm{T}$ cells become stimulated in the T-cell areas of the draining lymphoid tissues during the induction phase occurring soon after inoculation of the living bacteria (12). This T-cell stimulation can be evaluated in vivo qualitatively and quantitatively, by measuring the kinetics of the cells incorporating radiolabelled precursors of DNA (i.e. tritiated thymidine). But this global evaluation only partially reflects the clonal expansion of specifically engaged $\mathrm{T}$ cells. It was shown recently that, after intravenous injection of $\mathrm{BCG}$, among the radiolabelling present in the spleen suspension 10 or 20 days after BCG inoculation, only a small (25\%) but significant proportion of $\left[{ }^{3} \mathrm{H}\right]$ thymidine uptake was due to T cells (13). And amongst these radiolabelled $\mathrm{T}$ cells, only one tenth may represent specific committed T lymphocytes. In order to obtain a precise analysis of the number of cells engaged in protection, it is necessary to resort to a passive transfer of these cells in naive animals.

For instance, passive protection against tuberculosis but not leprosy can be evaluated in age and sex matched syngeneic recipient mice which had been rendered $\mathrm{T}$-cell deficient prior to transfer by exposure to $500 \mathrm{rad}$ of ionizing radiation (14).

As soon as these specific committed T-cells are stimulated, part of them leave the draining $\mathrm{T}$-cell areas via the lymphoid ducts through the systemic circulation and reach the inflammatory foci at the inoculation site or everywhere bacteria are present, and the host develops granulomatous reaction and macrophage activation. These reactions are elicited by specific recognition of antigens on the surface of infected macrophages by the circulating committed lymphocytes. these events stop further multiplication and dissemination of the mycobacteria. In addition, the host may also express a delayed-type hypersensitivity (DTH) reaction to mycobacterial antigens. usually, in the normal host, the development of this cellular hypersensitivity to mycobacterial antigens is associated with the occurrence of an acquired resistance to subsequent challenge of the same or related mycobacteria (15). Nonspecific immunity that has increased during mycobacterial infection can also be detected in vivo and in vitro by measuring the increase in resistance of the mononuclear phagocyte system against unrelated pathogen (i.e. Listeria monocytogenes), and adjuvant effect of DTH or Ab production to unrelated thymus-dependent antigens. This non-specific immunity can mask or modulate the real efficacy of the specific acquired resistance. However, such nonspecific resistance is not directly transferable in naive recipients, without the coinjection of mycobacterial antigens (14). All of these independent variables can be measured in the experimental animals in order to evaluate factors involved in the induction of the immune response after mycobacterial infections. But many of these variables are only correlates of pro- 
tective immunity, and thus only the transfer of immune $\mathrm{T}$ lymphocytes in challenged animals or in vitro microbicidal assays, may give the final results on protection.

Activated blood-borne macrophages accumulating in the newly formed tubercle seem to be the major cells involved in acquired rsistance against intracellular multiplying mycobacteria. However, the virulent tubercle bacilli may replicate within these cells or persist in a dormant state for a prolonged period. In has been shown that not all macrophages in a tubercule granuloma have the same function $(11,16)$. The subcellular mechanisms, which allow mycobacteria to resist intracellular killing in normal macrophages, and the nature of the bacteriostatic (rather than bactericidal) mechanisms observed within the phagolysosomes of activated macrophages, are still unknown. Evidence exists of the involvement of reactive oxygen (i.e. hydrogen peroxide $-\mathrm{H}_{2} \mathrm{O}_{2}$ ) in killing mycobacteria, and a good correlation was demonstrated between the capacity of a host macrophages to produce $\mathrm{H}_{2} \mathrm{O}_{2}$ after mycobacterial infection and the natural capacity, tested in vitro, of these macrophages to inhibit the growth of BCG (17). Experiments, with both human and mouse cells, in which the criteria for activation were the enhancing macrophage capacity of both to release $\mathrm{H}_{2} \mathrm{O}_{2}$ and kill protozoal and bacterial intracellular multiplying pathogens, give evidence that interferon gamma is the principal factor activating macrophages in infectious immunity $(18,19)$. Recently Douvas et al. (20) showed that IFN gamma stimulates human macrophages to become leishmanicidal, but $M$. tuberculosis grew better in these stimulated macrophages. It will clearly be important to discover which functional subsets are involved in mycobacterial killing and how mycobacterial products, antibodies and $\mathrm{T}$-cell products, influence the distribution of microorganisms between those subsets.

Thus, an important question remains: what are the nature and functions of the macrophages subsets in which the clinically relevant mycobacteria are present during infection and their relationship with immune T-cells (21). However, activation of macrophages alone may represent only one part of the acquird resistance mechanisms. Observations in guinea-pigs, mouse and man, show that distinct effector T-cell functions are not necessarily mediated by the same cells (11) and recent evidence shows, that in listeria infection, distinct murine $\mathrm{T}$-cell subsets were involved in promoting immune granuloma formation associated with systemic protection and those involved in locally induced protection and IFN gamma production (22). Thus increasing knowledge of the heterogeneity of antigens, lymphocytes, lymphokines and macrophages forces to reevaluate all experimental models used to explain the classical pathway of CMI to intracellular bacteria (11). The careful analysis of factors involved in induction of protective immunity to mycobacterial infection might help in such a way.

\section{Factors involved in induction of protective immunity}

Numerous models in different experimental animals have been used to evaluate protective immunity in mycobacterial diseases (23). At present, in vivo assays are more numerous and less discussed than in vitro assays. Mortality rate, evaluation of the kinetics of the growth of mycobacteria in the target organs and immunopathological reactions are the most used tests. However direct estimates of the viability of non-cultivable mycobacteria are always more time consuming because the organisms do not produce colonies in the normal way. usually, the total numbers of acid-fast bacilli (AFB) present in homogenates of whole organs are determined at varying time intervals after the challenge.

It has been claimed that if mixtures of live or dead bacteria were injected, the dead bacteria would have no obvious influence on growth of the live component (23). However, such an antigenic mass might influence regulatory mechanisms and, at least, modify the expression of the immune responses (24). Viability assays after intravenous injection of mice with $M$. leprae are not possible because growth in internal organs in very limited. However the 
bacilli grow in the mouse footpad and estimates of the proportion of viable bacilli present in suspensions of $M$. leprae and their doubling time can be made from the footpad growth curves (25). Uptake of a radio-labelled substrate, such as $\left[{ }^{3} \mathrm{H}\right]$ uracil or the metabolite of a radiolabelled substrate, have been proposed. But in order to obtain measurable uptake in reasonable time it is necessary to have a rather large number of viable bacilli, this restriction is one of the limits of the application for such methods.

The only natural «experiments» showing protection performed in man are the BCG vaccination trials against tuberculosis or leprosy. Results from such trials against tuberculosis, but also against leprosy, showed wide variations in protection, ranging from $0 \%$ to $80 \%$ (26). However the design inconcistencies and the epidemiological bias of such trials have been so numerous that interpretation is difficult. Nevertheless important points have emerged from these trials and from subsequent experimental studies: BCG vaccination has both succeeded and failed to protect simultaneously against tuberculosis and leprosy in different trials (27); BCG might fail in protecting against secondary tuberculosis in areas where it does protect against both disseminated phase of primary tuberculosis and leprosy (other than multibacillary cases). As already discussed, the first point is in agreement with the fact that protection from mycobacterial strain, as shown also in experimental models, can also be achieved by immunization with a different strain (28).

Such results support the hypothesis that common antigenic determinants possessed by different mycobacterial strains are relevant to the generation of protective cellular immunity and are either identical or closely cross reactive in various mycobacterial strains. However, the acquired resistance to mycobacteria or other intracellular multiplying bacteria appears to be critically dependent on immunizing with live rather than dead organisms (28). The basis for the requirement of living organisms for the induction of specific acquired antimycobacterial immunity is not yet understood. It may depend on the characteristics of the bacteria and host factors, since it is speculated that parasitization of host macrophages by live proliferative organisms may allow the presentation of so-called replication bacterial antigens to host $\mathrm{T}$ lymphocytes in a fashion which most efficiently induces immune protection.

\section{MYCOBACTERIAL FACTORS IN THE INDUCTION OF PROTECTIVE IMMUNITY}

Live BCG induces an effective antituberculosus immunity whether introduced into tissues by the intravenous, subcutaneous, intraperitoneal, aerogenic or oral route. Usually introduction of $10^{5}$ to $10^{6}$ viable BCG organisms through the IV route induces a maximal degree of protection against a virulent $M$. tuberculosis challenge given by the same route, 3 weeks after vaccination, but the presence of specific immune T cells can be detected as soon as after one week after immunization when higher doses of vaccine were given (13). No protection could be achieved when dead BCG in saline, whatever the dose given, was inoculated into mice (29). Moreover, not only live BCG need to be injected but also proliferating organisms need to be inoculated in order to induce protection. This evidence was demonstrated by a comparison between two BCG strains, one being streptomycin-susceptible, the other being streptomycin-resistant (SMY-res). Only the former was able to induce antituberculous resistance, the inability of the latter to immunize mice after IV injection was shown to be associated with a rapid inactivation of the in vivo population, so that viable counts in both liver and spleen decreased to subthreshold numbers, before significant degrees of antituberculous resistance can be induced (30). Subcutaneous injection of BCG-SMY-res did not induce any lymphoproliferative response in the draining lymph node (24). Thus it seems that only live and proliferative BCG strains, able to resist non-specific resistance, are able to induce acquired protection. Moreover, evidence that IV injection of heat-killed mycobacteria, such as murine disseminating $M$. lepraemurium can trigger mechanisms which allow increased proliferation 
of a simultaneously live homologous inoculum (31), shows that killing does not have the same effect on immunogenicity for mice of all mycobacterial species (32). By contrast, human or armadillo derived irradiated and/or heat-killed $M$. leprae inoculation in mice is able to induce immune protection against homologous organisms (33) or against $M$. tuberculosis (34) or $M$. bovis BCG (35). Cloned T-cell lines were also obtained after in vivo immunization with irradiated $M$. leprae without any adjuvant (36). They exhibited helper type activity, producing interleukin-2, macrophage activating factor and interferon gamma, and were further characterized in terms of cross-reactivities with other species of mycobacteria. However, induction of cross protection against BCG infections by irradiated $M$. leprae lymph node cells occurred only in particular strains of mice (i.e. in C57B $1 / 6$ but not in C3H mice) (35), indicating that the immune response pattern evoked after mycobacterial infections, is determined partly by the organism used and partly by genetic factors of the host.

\section{HOST FACTORS IN INDUCTION OF PROTECTIVE IMMUNITY}

Experimental studies indicate clearly that protection from superficial infections differs from protection from systemic challenge, depending on the capacity of mycobacterial strains to disseminate and also on properties of the various mouse strains used. Also differences in the clinical course of tuberculosis and leprosy in human population and individuals have long been recognized (21). Recent associations with certain HLA antigens have suggested a close association with the expression of leprosy rather than susceptibility or resistance to the infective organism (37). It is clear from experimental studies that many genes interact to determine the level of resistance in an individual. Experimental infectious murine models with intracellular multipliying organisms using outbred, inbred, congenic, recombinant and mutant strains have been used in recent years in order to analyze such genetic control $(11,23)$. Hence studies with mycobacteria have shown that inbred and outbred mouse strains can be classified into those which are resistant and those which are susceptible. It was of interest to note about the results of such studies that strains, previously designated Ityr (Salmonella typhimurium resistant) and Lshr (Leishmania donovani resistant) were also resistant to intravenous challenge, with $M$. bovis (BCG) (38), or M. lepraemurium (39) and that strains designated Itys, Lshs, were susceptible. Resistance was shown to be dominant and was at least expressed through the control of a gene (or group of genes) located in the chromosome 1 . Although this gene(s) is not mapped, its expression seems to influence of both early multiplication of the organisms within the macrophages (40) and the later development of acquired resistance mechanisms $(38,41)$. In the case of the BCG study (38), differences in resistance were revealed mainly in the spleen, after three weeks of a low inoculum $\left(2 \times 10^{4}\right.$ viable $\mathrm{BCG} /$ mouse) of well dispersed bacilli (strain Montreal) inoculated intravenously. No such differences were observed in other organs or when higher doses were used. When BCG was inoculated subcutaneously, with various doses $\left(10^{4}\right.$ to $\left.10^{6}\right)$, growth curves kinetics showed no significant differences in resistance in the draining node or at the inoculum site, except at day 1 after challenge (41). Variations in differences of resistance were also noticed when various BCG vaccine strains or atypical mycobacteria were used, being related to the natural ability of such strains to replicate in the mouse (42). Usually low inocula of virulent, well dispersed BCG strain, injected intravenously into Bcgr mice showed little multiplication, whereas in Bcgs mice progressive growth occurred, peaking at three weeks and then declining. Subsequently lower numbers of viable bacilli were detected, for several weeks, in Bcgs than in Bcgr strains: this difference was more marked in liver and lungs and was dose dependent (fig. 1). 

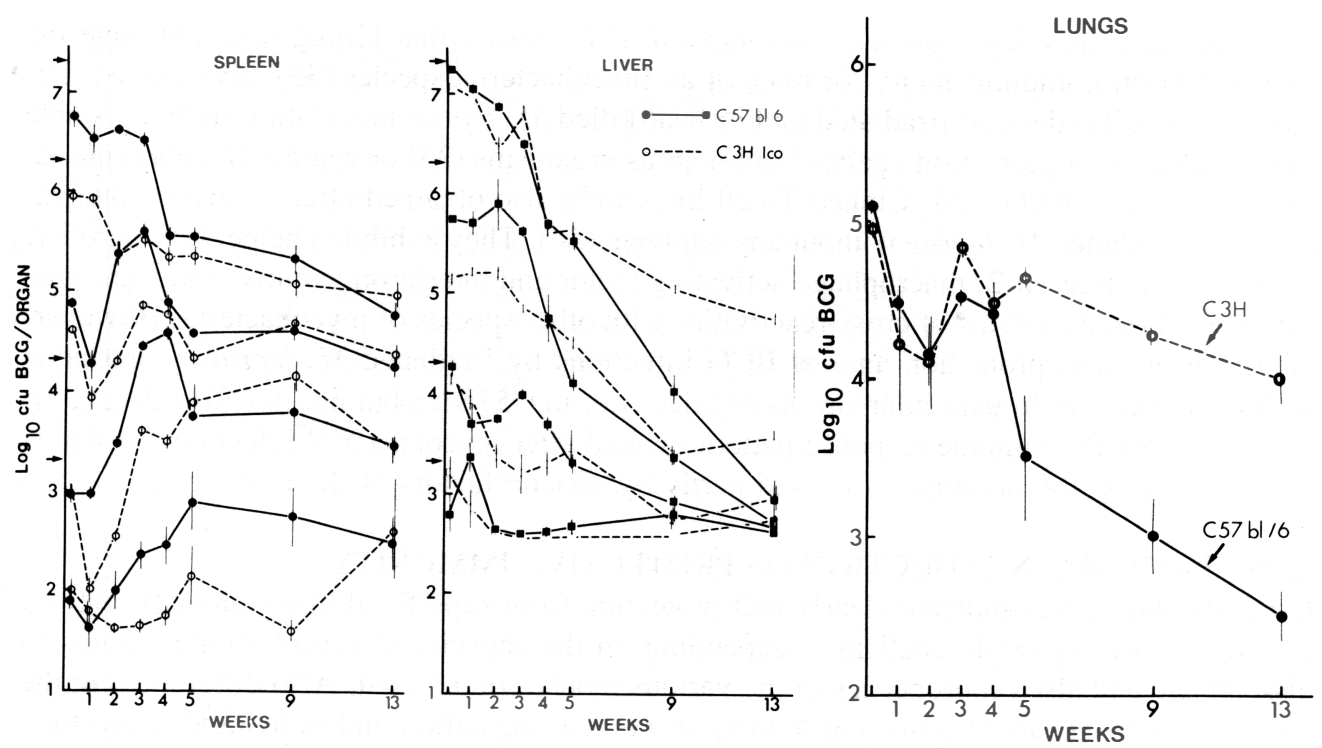

Figure 1:

Growth curves of BCG Pasteur (1173 P2) in spleen, liver and lungs of Bcgs (C57B 1/6 : closed symbol) and $\mathrm{Bcgr}$ (C3H/Ico : open symbol) mice injected intravenously with varying numbers of live organisms $-2 \times 10^{3}, 2 \times 10^{4}, 2 \times 10^{7} \mathrm{BCG}$ /mouse - (arrow). The representative growth curves in the lungs are those which were obtained with the highest dose of $B C G$ inoculated into the two strains of mice. Mean of 5 mice per group \pm SEM.

These results seem to indicate that the non-specific natural resistance in mice is quite able to prevent multiplication and persistance of a low inoculum of an attenuated living strain of $M$. bovis, without any help from a T-cell response. However, when a higher inoculum is given, $\mathrm{Bcgr}$ mice (i.e. $\mathrm{C} 3 \mathrm{H}$ ) were less able to prevent late multiplication and dissemination (in lungs). Classically it was shown that mice that had recovered from the primary infection developed DTH to tuberculin and showed strong resistance to further challenge with BCG or virulent tubercle bacilli (29), but when mice were inoculated IV with a low inoculum of BCG, only the Bcgs mice developed a 48 h DTH to tuberculin (43). Further, when several strains of mouse were inoculated subcutaneously with $4 \times 10^{6}$ BCG Pasteur and tested with tuberculin at varying periods of time after senitization, the time course patterns of such DTH reactions can be clearly separated into three groups, independently of the time after sensitization.

The results summarized in fig. 2, showed that for all mouse strains tested, except for Balb/c, a good correlation existed between natural susceptibility (Bcgs strains) and the protracted DTH type (type 3). 


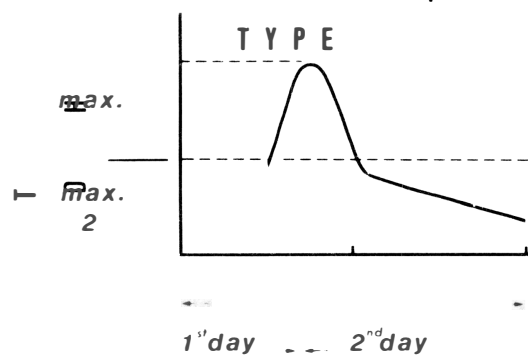

STRAIN DBA $/ 2^{\ominus}, A / J$

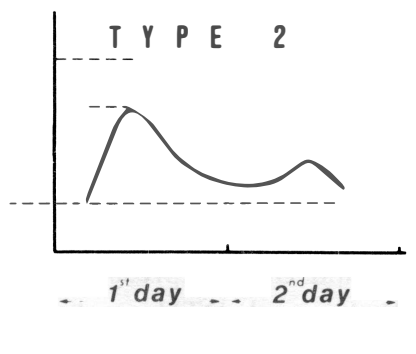

$$
\begin{gathered}
C 3 H^{\ominus}{ }_{\text {L.ab }}{ }^{\ominus}, A K R \\
C_{B A} \Theta_{B A L B / C}{ }^{\oplus}
\end{gathered}
$$

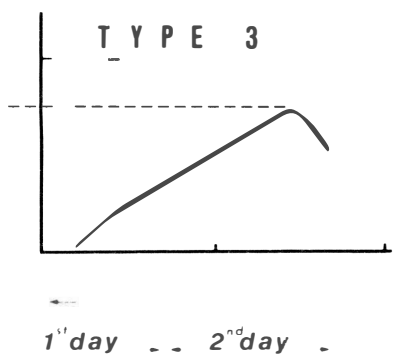

$C 57 B L \stackrel{\oplus}{\oplus}, \mathrm{H} . \mathrm{ab}^{\oplus}, \mathrm{DBA} / 1^{\oplus}$

Figure 2:

Schematical different time-courses of DTH reaction to tuberculin (4 $\mu \mathrm{g} / \mathrm{mouse})$ in $\mathrm{Bcg} \mathrm{g}^{\mathrm{s}}(+)$ and Bcgr (-) inbred mouse strains, immunized 14 days previously by one subcutaneous injection of $4 \times 10^{6}$ live BCG Pasteur into the left hind footpad. L.ab (low antibody) and H.ab (high antibody) mice are representative of mouse strains selected by Biozzi and coll.: in vivo curves of BCG Pasteur after a low inoculum given intravenously, showed the indicated Bc $g$ gene phenotype.

Type 1 and type 2 were observed in Bcgr strains. Acquired resistance after such immunization was then evaluated in Bcgs and Bcgr strains. Fourteen days after immunization, all mice, including controls, were inoculated IV with $10^{5}, 10^{6}$, or $10^{7}$ viable BCG and fourteen days later numbers of viable BCG were counted in spleens. The resistance index was calculated for each mouse strain, and compared to the tuberculin DTH level measured 18 or $42 \mathrm{~h}$ after elicitation, given on the challenge day in other gourp of mice used only for DTH measurements without any challenge.

\section{Figure 3:}

Acquired resistance to $B C G$ Pasteur, in mouse strains expressing the $18 \mathrm{~h}$ (left) or the 42 (right) DTH reactions to tuberculin. Separate groups of different inbred mouse strains were inoculated with one SC injection of $4 \times 10^{\circ}$ live $B C G$ and 14 days later, each group (including one control group per mouse strain) were either i.v. challenged with $1 \times 10^{5}, 1 \times 10^{6}$ or $1 \times 10^{7}$ live $B C G$, or $5 C$ inoculated in the contralateral footpad with $4 \mu \mathrm{g}$ tuberculin. For each tested strain, the resistance indexes were calculated from numbers of $\mathrm{CFU}$ found in spleen 14 days after the challenge in the normal control and in immune mice. The resistance indexes were then plotted against the peak level of the

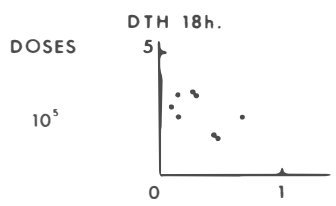

$10^{6}$

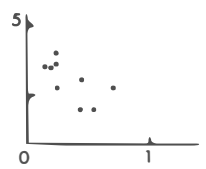

$10^{7}$

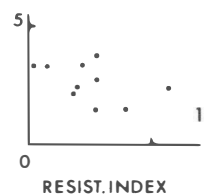

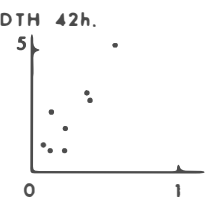
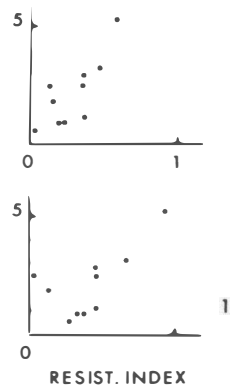
As shown in fig 3, a higher resistance index was found in strains presenting a protracted DTH type, and a good correlation was found between the resistance index and the DTH levels measured $42 \mathrm{~h}$ after elicitation. On the other hand no such correlation was found in strains of mouse presenting a $18 \mathrm{~h}$ reaction. This might be correlated with the fact that $\mathrm{Bcgr}$ mice had only a tenth of the number of granuloma in liver and spleen compared to the Bcgs mice (43).

The Balb/c mice constitute an exception from the observed correlation between Bcg gene expression (classified as Bcgs) and DTH expression, developing a type 2 DTH reaction. It was shown by Adu et al. (44) that such DTH reaction in Balb/c mice resembled the type 3 after 4 weeks, and there was no difference in the numbers of AFB recovered from the infected footpad between C57B1/6 and Balb/c mice during the periode of 7 days to 6 weeks. But it is difficult to draw conclusions from this, since AFB represent the total count of live and dead bacilli. When viable BCG was recovered from spleen in C57B1/6 and Balb/c mice, at varying intervals after an IV injection of a low inoculum of BCG Pasteur, no major difference between strains was observed during the first three weeks after challenge, but later a lower clearance rate was observed in Balb/c mice as compared with C57B 1/6 mice (Table 1).

\section{Table 1:}

Viable counts of BCG in spleens of $C 57 \mathrm{~B} 1 / 6$ and Balb/c mice measured at varying time intervals after an intravenous challenge of $1 \times 10^{+} B C G$.

\begin{tabular}{c|c|c}
\hline \multirow{2}{*}{ Days after challenge } & \multicolumn{2}{|c}{$\log _{10} \mathrm{CFU}$ of BCG/spleen \pm SEM $(\mathrm{N}=5)$} \\
\cline { 2 - 3 } & $\mathrm{C} 57 \mathrm{~B} 1 / 6$ & Balb/c \\
\hline 2 & $2.96 \pm 0.09$ & $3.04 \pm 0.04$ \\
9 & $3.10 \pm 0.05$ & $3.33 \pm 0.04$ \\
16 & $3.76 \pm 0.06$ & $3.64 \pm 0.16$ \\
23 & $4.43 \pm 0.04$ & $4.51 \pm 0.06$ \\
65 & $3.96 \pm 0.07$ & $4.28 \pm 0.08$ \\
70 & $3.41 \pm 0.08$ & $4.08 \pm 0.06$ \\
\end{tabular}

$\mathrm{a}=\mathrm{p}<0.05$ Student's test, compared to C57B1/6 infected groups.

Such low clearance rate was shown to be radiosensitive, since an increased delayed clearance rate occurred in Balb/c mice that had been sublethaly irradiated (500 rads) 24 hours before the IV injection of $1 \times 10^{4}$ viable BCG (fig. 4). 


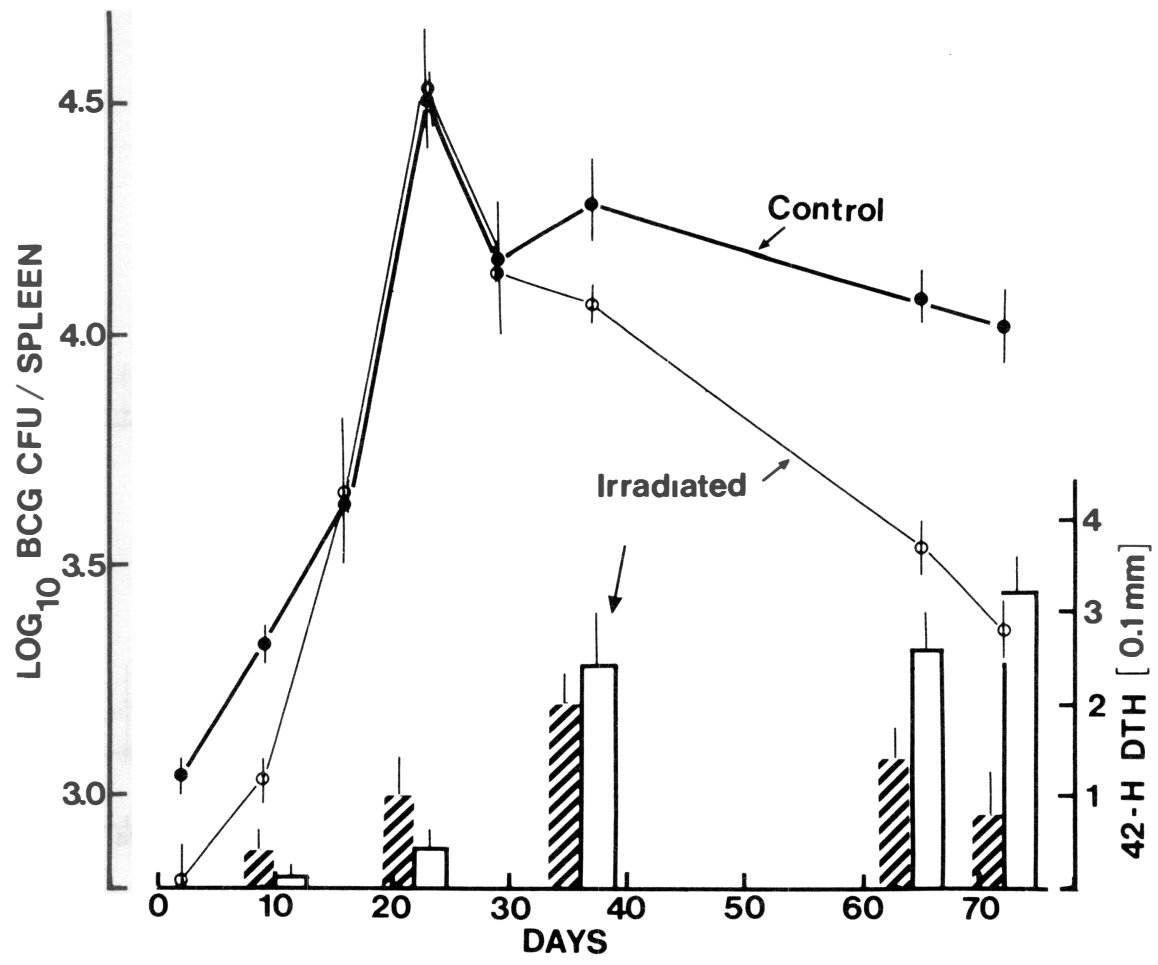

Figure 4:

Growth curves of BCG Pasteur in spleen of normal (closed symbol) and sublethaly irradiated (open symbol) Balb.c mice, inoculated intravenously with $1 \times 10^{-1}$ live BCG. The histogramms represent the tuberculin footpad reactivity (42h) following injection of $4 \mu \mathrm{g}$ tuberculin into the right hind footpad of separate groups of normal (hatched column) or $(500$ rads) irradiated (white bar) Balb/c mice inoculated with the same inoculum. Mean of 5 mice per group $\pm S E M$.

Moreover such infected preirradiated Balb/c mice expressed higher levels of the $42 \mathrm{DTH}$ reaction to tuberculin, as compared to infected normal Balb/c mice, and this occurred as they recovered from the primary infection.

No such radiosensitive phenomena were observed in other Bcgs or Bcgr strains of mouse tested (fig. 5). 

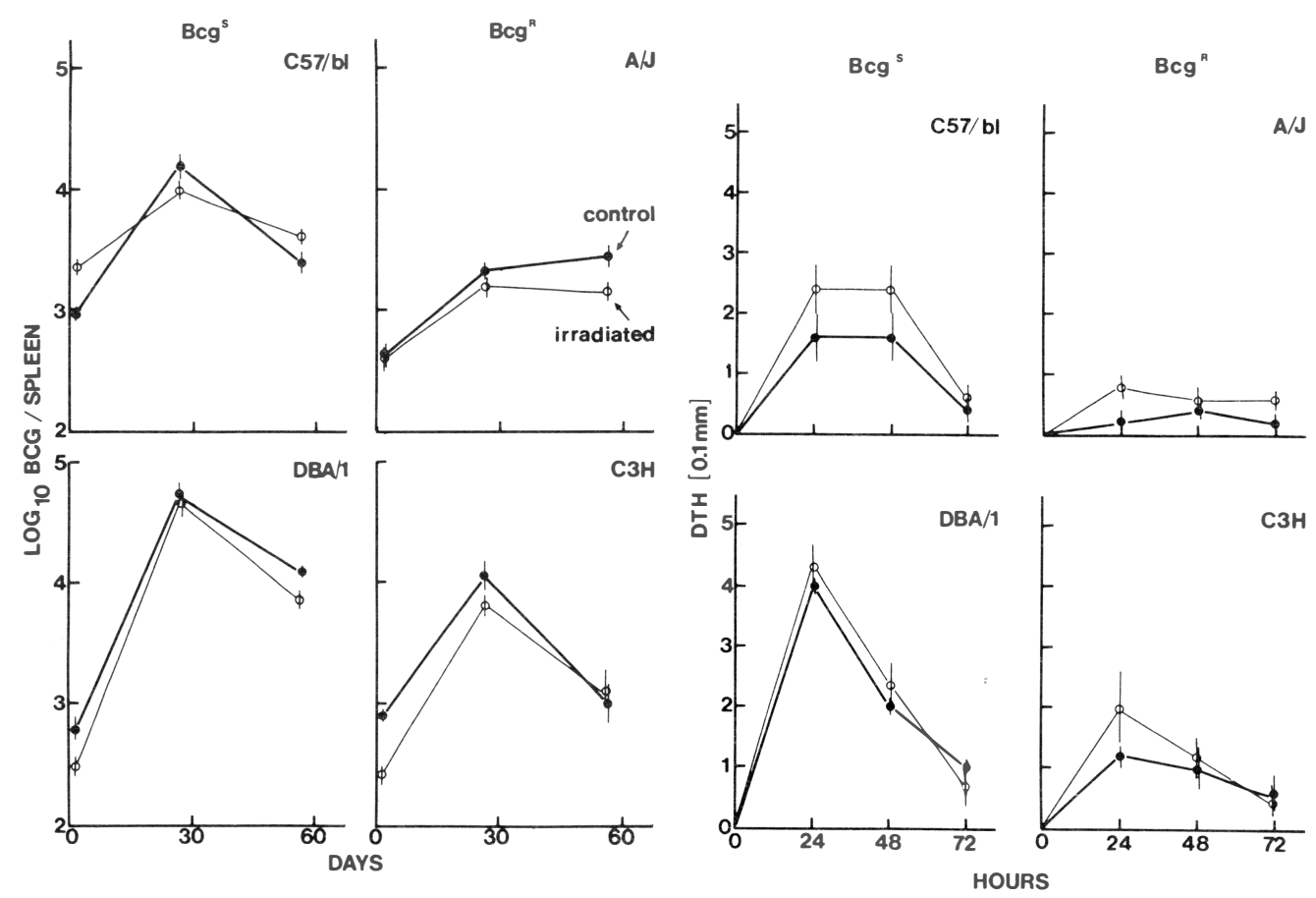

Figure 5:

Growth curves of BCG Pasteur in spleen (left) and day 56 DTH reactivity to tuberculin (4 $\mu$ g/mouse) (right) in normal (closed symbol) and sublethally irradiated (open symbol) Bcgs and Bcgr mouse strains, which were inoculated intravenously with $1 \times 10^{+}$live BCG. Mean of 5 mice per group \pm SEM.

Thus, the disappearance of the $42 \mathrm{~h}$ DTH reaction in Balb/c mice, associated with a low clearance rate after the primary infection might be due to the induction of an active particular subset of T-cells, with regulatory functions, observed here after a low inoculum of non-disseminating mycobacterial strain such as BCG. Similar results were also found after M. lepraemurium (45) and leishmania tropica (46) infections.

A nylon-wool adherent radiosensitive suppressor cell population in spleen was demonstrated in the case of $M$. lepraemurium infection, which was able to decrease resistance when injected in infected syngeneic mice. Thy $-1^{+}, \mathrm{L} 3 \mathrm{~T} 44^{+}$, and I-J phenotype cells, capable of inhibiting the induction and expression of DTH as well as reversing the healing of lesions were demonstrated in leishmanial infections. Such susceptibility to irradiation of this suppressor $\mathrm{T}$-cell population in those infections, including the BCG, was not found in other Bcgr strains of mouse. These suppressor $\mathrm{T}$ cells are thus different from those induced after a higher dose of BCG or after SC inoculation of M. lepraemurium (47). They might be triggered by different mechanisms either directly or indirectly through primary defects in macrophages or in a particular subpopulation of macrophages unable to present the protective antigen(s) to the right population of $\mathrm{T}$ lymphocytes. This hypothesis is now being tested in the laboratory. Thus depending upon the time, the multiplication, and the persistance of the microorganisms, accumulation of L3T4 lymphocytes will occur and function as suppressor cells, explicating non-healing lesions, dissemination, or low clearance rate after primary infection. The immune response may then vary from upgrading reactions (if L3T3 cells are prevented 
from functioning) to downgrading reactions (if mycobacteria continue to multiply and disseminate). Such variations in the immune response may resemble those observed in human borderline leprosy or in non-reactive intermediate tuberculosis. Other regulatory mechanisms have been described in $\mathrm{Bcg} s$ and $\mathrm{Bcg}$ r mouse strains, trying to explain acquired anergy, in vitro unresponsiveness and dissemination of microorganisms $(11,21,23)$. Usually they were produced after IV injection of high doses of non-disseminating mycobacteria (i.e. $\mathrm{BCG})$, or after subcutaneous injection of disseminating mycobacteria (M. lepraemurium, $M$. avium or $M$. intracellulare) in mice.

Lymphocyte trapping and induction of at least two types of regulatory suppressor cells ( $T$ lymphocytes and macrophages) may contribute to the nonspecific component of the defect, but these cells have never been shown to have any detrimental effect on the course of the infection, so they may be irrelevant to the immune protection mechanisms.

\section{Conclusions}

Conclusions drawn from experimental models of mycobacterial infections have been useful in the past in order to evaluate bacterial factors involved in the induction of CMI in general and peculiarly about protective immunity. Results obtained from BCG vaccine human trials on tuberculosis and/or leprosy, showing geographic variations in the acquired protection did lead to many hypotheses.

Two main hypotheses were developed during the sixties concerning the potency of various daughter BCG strains, and influence of previous sensibilization with atypical mycobacteria. However, careful analysis of such trials and experimental animal models did show that such hypotheses might not be involved in the results of the last trial, realized in south India $(26,28)$, since the 2 vaccines selected for the trial were the best selected strains, and that the only effect of atypical mycobacteria might be to immunize partially and then mask differences amongst vaccines and placebo vaccinated population, both being sensitized prior to vaccination. Thus other factors need to be looked out to explain differences in protection against tuberculosis and leprosy after BCG vaccination. Among such factors environmental and genetic factors might be involved concerning resistance and susceptibility to mycobacterial diseases.

Apart from the fact that nutritional and socioeconomic factors might be coagents in depressing immune responses and increasing contacts with pathogenic mycobacteria, interest in the genetic factors controlling immune responses after mycobacterial infections has increased in recent years. However, some confusion arose in literature concerning mostly the definition of the resistance or susceptibility in mouse models. In general, it results from the use of different species and strains of mycobacteria (without any definition of their virulence, ability or not to disseminate in the host), various inoculum doses, different routes of administration, different expressions of resistance, different patterns of CMI responsiveness or unresponsiveness. During the seventies, Smith gave an example of such situation: when several laboratories used animal test systems for evaluating vaccines against tuberculosis, no two laboratories were found using the same system (48). Further controlled studies of this situation showed that each major variable in animal test system, -such as species, vaccination route and dose, interval between vaccination and challenge, challenge route and dose-, contributed to induce a difference in ranking order of the effectiveness of a serie of live attenuated vaccines. If one adds variables concerning the host genetic factors, an increasing confusion is not surprising. Thus there is an urgent need for further in vivo and in vitro studies in several areas, primarily the existence and nature of the interrelationship between living mycobacteria and subpopulations of phagocytic mononuclear cells, and, further, the interaction between such infected subpopulations and the recruitment, clonal expansion and functions of 
the specific $\mathrm{T}$-cell subpopulations that arise after immunization. It will then be possible to proceed with the determination of the protective antigen, after having selected and cloned the right subpopulations of specific $\mathrm{T}$ cells capable of transferring efficient protection similar to that expected in humans.

\section{Acknowledgements}

This work was supported partly by the Immunology of tuberculosis (IMMTUB) of the UNDP/World Bank/WHO Special Program for Research and Training in Tropical Diseases. The authors thank Mrs J. Lortholary for typing the manuscript.

\section{References}

1 Strom RL, and Gruninger RP. AIDS with Mycobacterium avium intracellulare lesions ressembling those of Whipple's disease. N. Engl. J. Med., 1983, 309, 1323-1324.

2 Bloom BR. Learning from leprosy : a perspective on immunology and the third world. J. Immunol., 1986, 137, 1-10.

3 W.H.O., Immunological research in tuberculosis : memorandum from a WHO meeting. Bull. WHO, 1982, 60, 723-727.

4 Reggiardo Z. and Middlebrook G.. Failure of passive serum transfer of immunity against aerogenic tuberculosis in rabbits. Proc. Soc. Exp. Biol. Med., 1974, 145, 173-175.

5 Forget A, Benoit JC, Turcotte R. and Gusew-Chartrand N.. Enhancement activity of antimicrobacterial sera in experimental Mycobacterium bovis (BCG) infection in mice. Infect. Immun., 1976, 13, 1301-1306.

6 Forget A, Turcotte R, Benoit JC. and Borduas AG. Enhancing effect of passive immunization with mycobacterial antibodies on humoral and cellular immunity in BCG-infected mice. Ann. Immunol. (Inst. Pasteur), 1978, 129G, 255-265.

7 Hahn H, and Kaufmann SHE. The role of cell-mediated immunity in bacterial infections. Rev. Infect. Dis., 1981, 3, 1221-1250.

8 Chaparas SD. The immunology of mycobacterial infections. C:R:C: Crit. Rev. Microbiol., 1982, 9, 139-197.

9 Collins FM. The immunology of tuberculosis. Amer. Rev. Resp. Dis., 1982, 25 (suppl. 3), 42-49.

10 Daniel TM. The immunology of tuberculosis, Clin. Chest. Med. 1980, 1, 189-201.

11 Rook GAW. An integrated view of the immunology of the mycobacterioses in guineapigs, mice and men. In Biology of Mycobacteria. vol. 2. C. Ratledge and JL Stanford Eds. Acad. Press. London, 1983, 279-319.

12 Hawrylko E. and Mackaness GB. The kinetics of lymphoid proliferation in tuberculosis mouse spleen cells. Cell. Immunol. 1972, 5, 148-170.

13 Orme IM, Ratcliffe MJH and Collins FM. Acquired immunity to heavy infection with Mycobacterium bovis, bacillus Calmette-Guérin, and its relationship to the development of non specific unresponsiveness in vitro. Cell. Immunol., 1984, 285-296.

14 Lefford MJ. Transfer of adoptive immunity to tuberculosis in mice. Infect. Immun., $1975,11,1175-1181$.

15 Lefford MJ. Induction and expression of immunity after BCG immunization. Infect. Immun., 1977, 18, 646-653.

16 Suga M, Dannenberg AM and Higuchi S. Macrophage functional heterogeneity in vivo. Amer. J. Path., 1980, 99, 305-324.

17 Stach JL, Delgado G, Tchibozo V, Strobel M, and Lagrange PH. Natural resistance to mycobacteria : antimicrobial activity and reactive oxygen intermediate releasing function of murine macrophages. Ann. Immunol. (Inst. Pasteur), 1984, 135D, 25-37. 
18 Nathan CF, Murray HW, Wiebe ME, and Rubin BY. Identification of interferon gamma as the lymphokine that activates human macrophage oxidative metabolism and antimicrobial activity. J. Exp. Med., 1983, 158, 670-689.

19 Kiderlen AF, Kaufmann SHE, and Lohmann-Matthes ML. Protection of mice against the intracellular bacterium Listeria monocytogenes by recombinant immune interferon. Eur. J. Immunol., 1984, 14, 964-967.

20 Douvas GS, Lookes DL, Vatter AE and Crowle AJ. Gamma interferon activates human macrophages to become tumoricidal and leismanicidal but enhances replication of macrophages associated Mycobacteria. Infect. Immun., 1985, 50, 1-8.

21 Lagrange PH, and Hurtrel B. Anergy and other immunological perturbances in mycobacterial infections : an overview. In Tuberculosis : interactions with the immune system ; H. Friedman and M. Bendinelli, Eds, Plenum publ. co., New-Yok, in press.

22 Näher $\mathrm{H}$, Sperling U, and Hahn $\mathrm{H}$. $\mathrm{H}_{2} \mathrm{k}$ restricted granuloma formation by Ly2+ T-cells in antibacterial protection to facultative intracellular bacteria. J. Immunol., 1985, 135, 569-572.

23 Brown IN. Animal models and immune mechanisms in mycobacterial infections. In Biology of Mycobacteria, (C. Ratledge and JL. Stanford, eds) Acad. Press. London, vol. 2, 1983, 173-234.

24 Mackaness GB, Auclair DJ, and Lagrange PH. Immunopentiation with BCG: 1. Immune response to different strains and preparations. J. Nat. Cancer Inst., 1973, 51, 1655-1667.

25 Shepard CC, and Mc Rae DH. A method for counting acid fast bacteria Int. J. Lepr., 1968, 36, 78-82.

26 Lagrange PH, Hurtrel B, Brandely M, and Thickstun PM. Immunological mechanisms controlling mycobacterial infections. Bull. Europ. Physiopath. Resp., 1983, 19, 163-172.

27 Rook GAW, Bahr GM, and Stanford JL. The effects of two distinct forms of cell-mediated response to mycobacteria on the protective efficacy of BCG. Tubercle, 1981, 62, 63-68.

28 Orme IM, and Collins FM. Cross protection against non-tuberculous mycobacterial infections by Mycobacterium tuberculosis memory immune T-lymphocytes. J. Exp. Med., 1986, 163, 203-208.

29 Collins FM. Acquired resistance to mycobacterial infections. Adv. Tuberc. Res., 1972, 18, $1-30$.

30 Collins FM, and Montalbine V. Relative immunogenicity of streptomycin-susceptible and resistant strains of BCG : II- Effect of the route of immunization on growth and immunogenicity. Amer. Rev. Resp. Dis., 1975, 111, 43-51.

31 Lagrange $\mathrm{PH}$, and Hurtrel B. Local immune response to Mycobacterium lepraemurium in C3H and C57B1/6 mice. Clin. Exp. Immunol., 1979, 461-474.

32 Orme IM, and Collins FM. Infection with Mycobacterium kansaii and efficacy of vaccination against tuberculosis. Immunology, 1983, 50, 581-586.

33 Shepard CC, Walker L, and Van landingham RM. Heat stability of Mycobacterium leprae immunogenicity. Infect. Immun., 1978, 22, 87-93.

34 Patel PJ, and Lefford MJ. Specific and non specific resistance in mice immunized with irradiated Mycobacterium leprae. Infect. Immun., 1978, 20, 692-697.

35 Lagrange PH, Hurtrel B, Ravisse P, and Grosset J. A single subcutaneous inoculation of $10^{7}$ armadillo derived irradiated Mycobacterium leprae evokes different immunological behavior in C57bl/6 and C3H mice. Ann. Microbiol. (Inst. Pasteur), 1982, 133B, 167-168. 
36 Kingston AE, Stagg AJ, and Colston MJ. Investigation of antigen cross-reactivity of $M y$ cobacterium leprae reactive murine T-cell lines and clones. Immunology, 1986, 58, 217-223.

37 Van Edden W, Gonzalez NM, De Vries RRP, Convit J, and Van Rood JJ. HLA linked control of predisposition to lepromatous leprosy. J. Infect. Dis., 1985, 151, 9-14.

38 Gros P, Skamene E, and Forget A. Genetic control of natural resistance to Mycobacterium bovis (BCG) in mice. J. Immunol., 1981 127, 2417-2431.

39 Brown IN, Glynn AA, and Plant JE. Inbred mouse strain resistance to Mycobacterium lepraemurium follows the Ity/Lsh pattern. Immunology, 1982, 47, 149-156.

40 Stach JL, Gros P, Forget A, and Skamene E. Phenotypic expression of genetically controlled natural resistance to Mycobacterium bovis. J. Immunol., 1984, 132, 888-892.

41 Hurtrel B, Hurtrel M, and Lagrange PH. Genetic control of tuberculin time course in mice, correlation with natural and acquired resistance against BCG. In Genetic control of host resistance to infection and malignancy. (E. Skamene, Ed), A.R. Liss Inc., NewYork, 1985, 305-312.

42 Denis M, Forget A, Pelletier M, Turcotte R, and Skamene E. Control of the BCG gene of early resistance in mice to infection with BCG substrains and atypical mycobacteria. Clin. Exp. Immunol., 1986, 63, 517-525.

43 Pelletier M, Forget A, Bourassa D, Gros P, and Skamene E. Immunopathology of BCG infection in genetically reistant and susceptible mouse strains. J. Immunol., 1982, 129, 2179-2185.

44 Adu HO, Curtis J, and Turk JL. Differences in cell-mediated immune responses of «high-resistance» and «low-resistance» mice to a non pathogenic mycobacterium. Scand. J. Immunol., 1981, 14, 467-480.

45 Alexander J. Adoptive transfer of immunity and suppression by cells and serum in early Mycobacterium lepraemurium infections in mice. Parasite Immunol., 1979, 1, 159-166.

46 Howard JG, Hale C, and Liew FY. Immunological regulation of experimental cutaneous leishmaniasis. IV. Prophylactic effect of sublethal irradiation as a result of abrogation of suppressor T-cell generation in mice gentically susceptible to Leishmania tropica. J. Exp. Med., 1981, 153, 557-568.

47 Rook GAW. Supressor cells of mouse and man - What is the evidence that they contribute to the eatiology of the mycobacterioses? Lepr. Rev., 1982, 53, 306-312.

48 Smith DW. Animal models for study immunity in infection disease. J. Infect. Dis., 1973, 128, 800-801. 